\title{
Development of an Electrochemical Nano-biosensor for Rapid and Sensitive Diagnosis of Bilharzia in Kenya
}

\author{
Joseph Odundo ${ }^{a}$, Naumih Noah ${ }^{b, *(\mathbb{D})}$, Dickson Andala ${ }^{c}$, Janet Kiragu ${ }^{a}$ and Eric Masika ${ }^{a}$ \\ ${ }^{a}$ Department of Chemistry, Kenyatta University, P.O Box 43844-00100, Nairobi, Kenya. \\ ${ }^{b}$ School of Pharmacy and Health Sciences, United States International University-Africa, P.O Box 14634-00800, Nairobi, Kenya. \\ ${ }^{c}$ Department of Chemistry, Multimedia University of Kenya, P.O Box 30305-00100, Nairobi, Kenya.
}

Received 22 January 2018, revised 21 September 2018, accepted 5 October 2018.

\begin{abstract}
Bilharzia, also known as schistosomiasis, is still affecting more than 250 million people, killing about 200000 annually and leaving millions of others debilitated. Current diagnostic tools have extremely low validity and are not readily applicable for rapid diagnosis in rural and remote areas. In low-grade infections, microscopy, the most commonly used method, may have low sensitivity. For example, in suspected cases, up to five real sample specimens are recommended for increased sensitivity. The rise of nanotechnology has created the potential to significantly improve the current diagnostic approaches. Conjugating antibodies with nanoparticles pools the distinctive properties of the nanoparticles with the specificity and selectivity of the antibodies in the recognition of the antigen. This in return leads to a very versatile and specific mixed product with improved analytical signal which is important for fast, sensitive and real-time point-of-care diagnosis. In this work, we report the use of nanotechnology to develop a simple and sensitive nano-biosensor which consisted of a nano-strip with immobilized gold nanoparticles conjugated with bilharzia antibody, and demonstrated its potential for diagnosis of bilharzia antigen. A detection range of $1.13 \times 10^{1} \mathrm{ng} \mathrm{mL}^{-1}$ to $2.3 \times 10^{3} \mathrm{ng} \mathrm{mL}^{-1}$ of bilharzia antigen in stool samples and with a limit of detection of $8.3887 \times$ $10^{-2} \mathrm{ng} \mathrm{mL}^{-1}$ was attained, indicating the potential of the nano-biosensor to be used to detect bilharzia antigen in stool samples.
\end{abstract}

KEYWORDS

Bilharzia, nanobased-biosensor, nanostrips, nanokits.

\section{Introduction}

Bilharzia, also known as schistosomiasis, is one of the most neglected tropical diseases (NTDs), yet it is still affecting more than 250 million people, killing about 200000 annually and leaving millions others debilitated, especially in the remote rural populations. ${ }^{1}$ Bilharzia has a negative direct impact on health. In addition, it also fuels the vicious circle of poverty and stigma that leaves people unable to work, go to school, or participate in family and community life. ${ }^{1}$ It is caused by the schistosome parasite $^{2}$ and in human, it is caused by S. haematobium, S. mansoni, S. japonicum and less frequently by S. mekongi and S. intercalatum. The infection with cercariae arises when the skin gets into contact with infested water. ${ }^{3 \mathrm{~b}-\mathrm{d}}$

Diagnosis is a key step in the treatment and control of this parasitic disease, since case detection, morbidity assessment and the evaluation of control strategies all build from the diagnostic results. The current diagnostic methods for bilharzia include the microscopic determination of parasite eggs either in urine or stool or by antibody-antigen detection methods. ${ }^{4}$ In addition, the sensitivity of the diagnosis also depends on the severity of the infection. For example, in low-grade infections, the sensitivity of one microscopic examination may be very low and when bilharzia is suspected up to five urine or stool specimens are recommended for microscopic analysis to improve the sensitivity of the examinations. ${ }^{5}$ Moreover, based on the method and the time used in the post-infected host, the current antibody assays report a sensitivity in the range of between 65 and $86 \%{ }^{5}$ Some commonly used methods such as the ones based on the

* To whom correspondence should be addressed. E-mail: mnoah@usiu.ac.ke detection of antibodies against the soluble egg antigen (SEA) may lead to the retention of the eggs and constant secretion of the SEA by the deposited egg, hence the antibodies may be elicited for an indefinite period of time after the primary infection, irrespective of successful treatment. These methods thus suffer from low sensitivity ${ }^{6}$ and in addition, they often give unreliable results especially in areas of prevalence. ${ }^{7}$

Nanotechnology, which has the potential to offer not only improvement to current approaches, but also unexpectedly delivers many new tools and capabilities, ${ }^{8}$ can be used to improve the sensitivity of these methods. The application of metal nanoparticles in immune-sensing has shown great potential in developing versatile point-of-care diagnostic devices which are highly sensitive. ${ }^{9}$ This is due the small size of the metal nanoparticles (NPs) which makes their physical and chemical properties exceptional as compared to their corresponding bulk materials. Gold nanoparticles (AuNPs) for instance, have drawn widespread application in biomedical diagnostic assays and for imaging ${ }^{10}$ due to their biocompatibility, high stability and ease of characterization. For example, AuNPantibody conjugates have been used in immunohistochemistry, biosensors and immunoassays as either optical or electrochemical biomarkers. ${ }^{11}$ AuNPs have also been attached to protein molecules for drug delivery targeting cancer cells, ${ }^{12}$ attached to glucose oxidase for glucose biosensing ${ }^{13}$ and to antibodies for spectro-recognition. ${ }^{14}$

Conjugating antibodies with nanoparticles pools the distinctive properties of the nanoparticles with the specificity and selectivity of the antibodies in the recognition of the antigen. ${ }^{15}$ 
This results in a highly versatile and specific hybrid product with improved analytical signal important for rapid, sensitive and real-time point-of-care diagnosis. The need to perform rapid 'in situ' analyses has seen the use of disposable screen-printed electrodes (SPEs) replace the conventional electrodes. ${ }^{16}$ Due to the heterogenic nature of the screen-printing technology together with the properties of solid electrodes, SPEs have numerous benefits as compared to others for industrial sensor production. ${ }^{17}$ For example, gold SPEs may be coated with additional layers by again screen-printing, adsorption, electropolymerization, etc., ${ }^{17-18}$ which can allow for automated, rapid and point-of-care application.

In this work, we have used nanotechnology to develop a simple and sensitive nano-biosensor for detection of bilharzia antigen (SEA) using gold screen-printed electrodes and demonstrated their potential for diagnosis of bilharzia antigen. The objective of this study therefore was to develop a versatile device for the diagnosis of SEA with a low detection limit. Kenya depends on agricultural farming where bilharzia is prevalent in rice-growing areas such as Mwea, Coast and Nyanza. This eventually affects the economy since many people are unable to go to work and even school-going children may not be able to attend school. This nano-biosensor can be fabricated to nanokits using the screen-printed electrodes as strips which can be distributed to bilharzia endemic areas in Kenya like Nyanza and Mwea regions to facilitate early diagnosis and treatment of bilharzia.

\section{Materials and Methods}

\subsection{Reagents}

Dimethylaminopropyl-N'Ethylcarbodiimide N-3 hydrochloride (EDC/EDC-HCl) and $\mathrm{N}-\mathrm{Hyd}$ droxy succinimide (NHS) were purchased from Fluka. Sodium phosphate monohydrate, Sodium phosphate dihydrate, sodium chloride and sodium hydrogen carbonate of analytical grade, monodispersed $10 \mathrm{~nm}$ $\mathrm{Au}$ nanoparticles, stabilized in $0.1 \mathrm{mM}$ PBS and reactantfree were obtained from Sigma Aldrich (St. Louis, MO, USA). bovine serum albumin (BSA) was purchased from Thermo Fischer Scientific (Pittsburgh, PA). Rabbit anti-schistosome and mouse schistosome-SEA were prepared at the Kenya Medical Research Institute (KEMRI), Nairobi, Kenya. Infected human faecal samples were obtained from Kandongu Health Dispensaries in Mwea Rice Irrigation scheme, Kirinyaga County, Kenya.
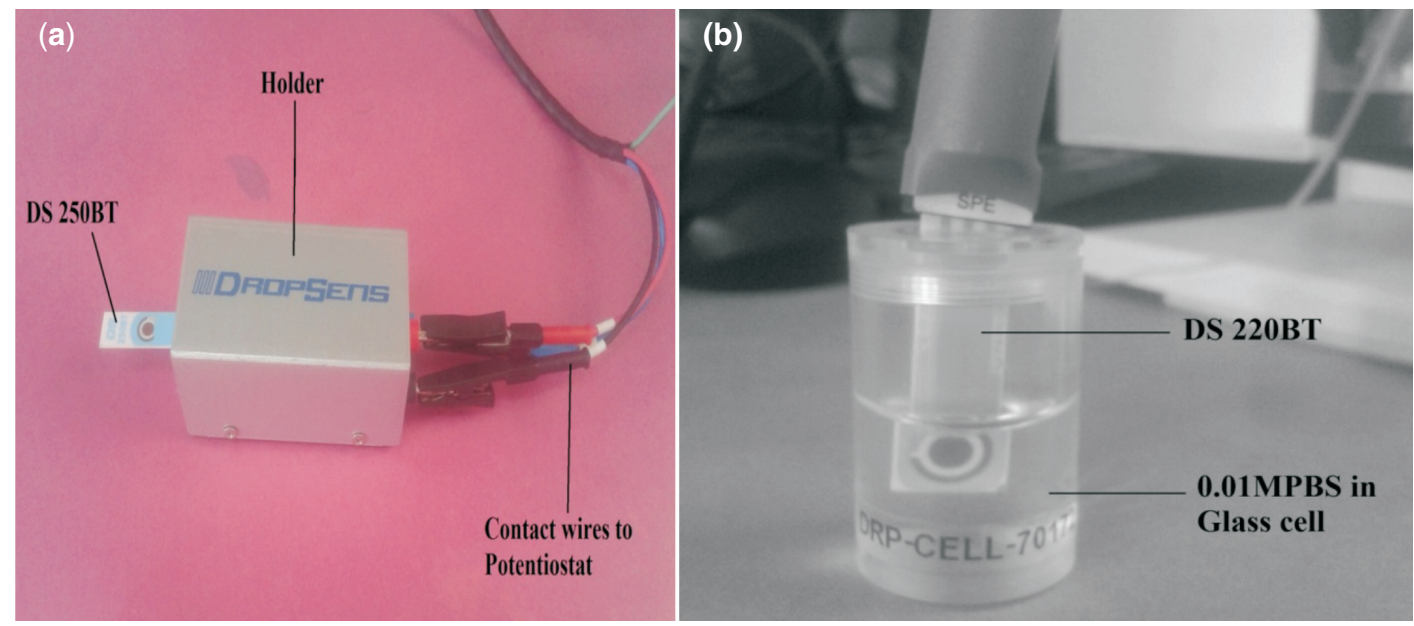

Figure 1 Representative set-up for electrochemical study of the Ab-Ag interaction using low temperature screen-printed electrodes (a) SPE model DS250BT and (b) SPE model DS220BT.
2.2. Preparation of SEA and Anti-schistosoma Antibody (IgG) This procedure was done at the Centre for Biotechnology Research and Development (CBRD) at KEMRI, Kenya, and is reported in the literature..$^{19}$ Briefly, Schistosoma mansoni parasite eggs were perfused from the liver and gut of infected mice, crushed and ground in pre-cooled mortars. The supernatant obtained after centrifuging was collected and concentrated to obtain SEA. Adult rabbits were injected with SEA and the serum extracted 6 weeks post-injection to obtain the antibody. The concentration of the antibody was determined using direct ELISA. ${ }^{20}$

\subsection{Nano Biosensor Procedures}

\subsubsection{Instrumentation}

The biological interaction of anti-schistosome with the SEA was investigated using a BASi Epsilon Potentiostat (Electrochemical Workstation, Version 2.13.77, USA). The low temperature gold screen-printed electrodes obtained from Dropsens, Spain, were connected to the potentiostat via a holder (DS 250BT) or a glass cell (DS 220BT) as shown in Fig. 1a,b. The conjugate was immobilized on the working electrode of SPE. The scans were done in triplicate and the controls involved running the sensor without the analyte.

\subsubsection{Preparation of Gold Nanoparticle Anti-bilharzia Conjugate (AuNP-IgG) Conjugate}

The gold nanoparticle anti-bilharzia conjugate was prepared as reported in literature. ${ }^{19}$ Briefly, an aliquot of rabbit antibilharzia IgG (28.9 $\left.\mathrm{mg} \mathrm{mL}^{-1}\right)$ stock solution was diluted with $0.01 \mathrm{M}$ PBS (pH 7.4) to $10 \mu \mathrm{lmL}^{-1}$. A solution containing a mixture of $1 \mathrm{~mL}$ of $0.1 \mathrm{M}$ EDC and $1 \mathrm{~mL}$ of $0.4 \mathrm{M}$ NHS was prepared in $5 \mathrm{~mL} 0.01 \mathrm{M}$ PBS. $50 \mu \mathrm{l}$ of $10 \mathrm{~nm}$ AuNPs stabilized in $0.01 \mathrm{M}$ PBS was added to the mixture followed by $10 \mu \mathrm{l}$ of $10 \mu \mathrm{g} \mathrm{mL}^{-1}$ rabbit anti-bilharzia. This was gently stirred for half an hour at room temperature. The suspension was collected via centrifugation at $3500 \mathrm{rpm}$ for $1 \mathrm{~h}$. The supernatant was discarded and the suspension washed twice with $0.01 \mathrm{M}$ PBS before re-suspending it in the buffer solution and kept at $4{ }^{\circ} \mathrm{C}$. This procedure was then repeated two more times.

\subsubsection{Optimization of AuNP-IgG Conjugate}

The sensor was optimized for several parameters which included concentration of reagents used in the preparation of the conjugate, time of incubation (assay time) and ratio of 
EDC:NHS used. The optimization was achieved by carrying out the experiments with different concentrations of the reagents and at various assay times. The optimum operating conditions were determined by comparing the signals obtained for the analysis and the assay time.

\subsubsection{Immobilization of the AuNP-IgG Conjugate on the Sensor Platform}

The sensor set-up involved the immobilization of an optimized gold nanoparticle-anti-bilharzia conjugate (AuNP-IgG) on the working electrode, a gold-coated screen-printed electrode as shown in Scheme 1. Briefly, $1.0 \mu \mathrm{g} \mathrm{mL}^{-1}$ conjugate was prepared and $10 \mu \mathrm{limmobilized} \mathrm{on} \mathrm{the} \mathrm{working} \mathrm{electrode} \mathrm{of} \mathrm{the}$ two types of gold ink SPEs (DS 250BT and DS 220BT) for $1 \mathrm{~h}$. This was followed by washing with phosphate buffer with Tween-20 (PBST) and then deactivated with $1 \%$ BSA for 15 min to block any free unbound sites of the antibody. The procedure was done in triplicate. The two models of gold SPEs used in the study were set as shown in Fig. 1a,b. The SPEs were then characterized using used cyclic voltammetry $(\mathrm{CV})$ and differential pulse voltammetry (DPV).

\subsubsection{Rabbit Anti-schistosome and Mouse Schistosome SEA Interaction}

Different concentrations of Mouse Schistosome SEA $0.05-50 \mathrm{ng} \mathrm{mL}^{-1}$ were prepared in 0.01M PBS $(\mathrm{pH}=7.6)$ buffer and used as the analyte for the interaction of the immobilized rabbit anti-schistosome antibody which was conjugated to AuNPs. The sensor was incubated in SEA for a fixed period of $1 \mathrm{~h}$. This procedure was done in triplicate. The biological recognition that arises from the Ab-Ag interaction brought about changes in the electrochemical activity of the nanoparticles on the surface of the electrode. These changes were then characterized using CV and DPV. The CV scans were done at a scan rate of $50 \mathrm{mV} \mathrm{s}^{-1}$ between 0 and $1.5 \mathrm{~V}$ whereas DPV was ran between $-0.1 \mathrm{~V}$ and $-0.5 \mathrm{~V}$, pulse amplitude $50 \mathrm{mV}$ and pulse width $50 \mathrm{~ms}$.

\subsection{Preparation of Faecal Samples for Analysis and}

Determination of SEA in the Real Samples

$100 \mathrm{mg}$ of the sample was diluted with $400 \mu \mathrm{l}$ of $0.01 \mathrm{M}$ PBS. The mixture was vortexed for $5 \mathrm{~min}$ to loosen the $S$. mansoni eggs and release the miracidia. The mixture was then decanted to separate the insoluble material and large debris. Clearer liquid part of the sample was obtained by centrifugation at $3000 \mathrm{rpm}$ for $1 \mathrm{~h} .100 \mu \mathrm{l}$ of the supernatant was carefully drawn, labelled and stored for analysis at $4{ }^{\circ} \mathrm{C}$. The pellet was discarded. To investigate the workability of the sensor, the supernatant obtained from the infected human faecal material was diluted to a factor 4 using $0.01 \mathrm{M}$ PBS buffer. This was then analyzed using the same procedure used for the standards of SEA above. The real concentration of the analyte in the sample was then obtained from the calibration curve for the SEA standards.

\section{Results and Discussion}

\subsection{Preparation and Characterization of the Nanoparticle-} anti-bilharzia Conjugate

The characterization of the nanoparticle-anti-bilharzia conjugate was done using UV/Vis and CV. The UV/Vis results as shown in Fig. 2a indicate a reduction in the peak absorbance of AuNPs in the conjugate as compared to the bare nanoparticles. ${ }^{19}$ This was as a result of the steric hindrance brought about by the antibodies that are covalently bonded on the surface of the nanoparticle during the formation of the conjugate. This hindrance reduces the exposure of AuNPs to the incident light, thus reduced oscillation of electrons in the conduction band. ${ }^{22}$ Furthermore, the peak is observed to appear at the same wavelength $(640 \mathrm{~nm})$, an indication of the stability of the conjugate so formed. ${ }^{23}$ The zero valent cross-linker EDC-HCl is known to react with carboxylated AuNPs in the presence of NHS to form amine-reactive NHS esters. Subsequent addition of antibilharzia antibodies results in coupling between the AuNPs and primary amines on the antibody via stable amide bonding. ${ }^{19}$

The electrochemical characterization of the nanoparticle-antibilharzia conjugate was done using cyclic voltammetry $(\mathrm{CV})$. One of the major features in CV is its ability to provide information about the chemistry of redox couples. Electroactive compounds give peaks at the oxidation and reduction potentials which are characteristic of the cation undergoing the redox process. As shown in Fig. 2b, CV results ${ }^{19}$ indicated a decrease in the peaks for the nanoparticle-anti-bilharzia conjugate as compared with the peaks for the AuNPs alone. This indicated an insulation of the active part of the electrode by the conjugate since it is not electroactive. No peak was observed with the antibody alone implying that the protein molecule is not electroactive and therefore the reduction of the anodic currents proves

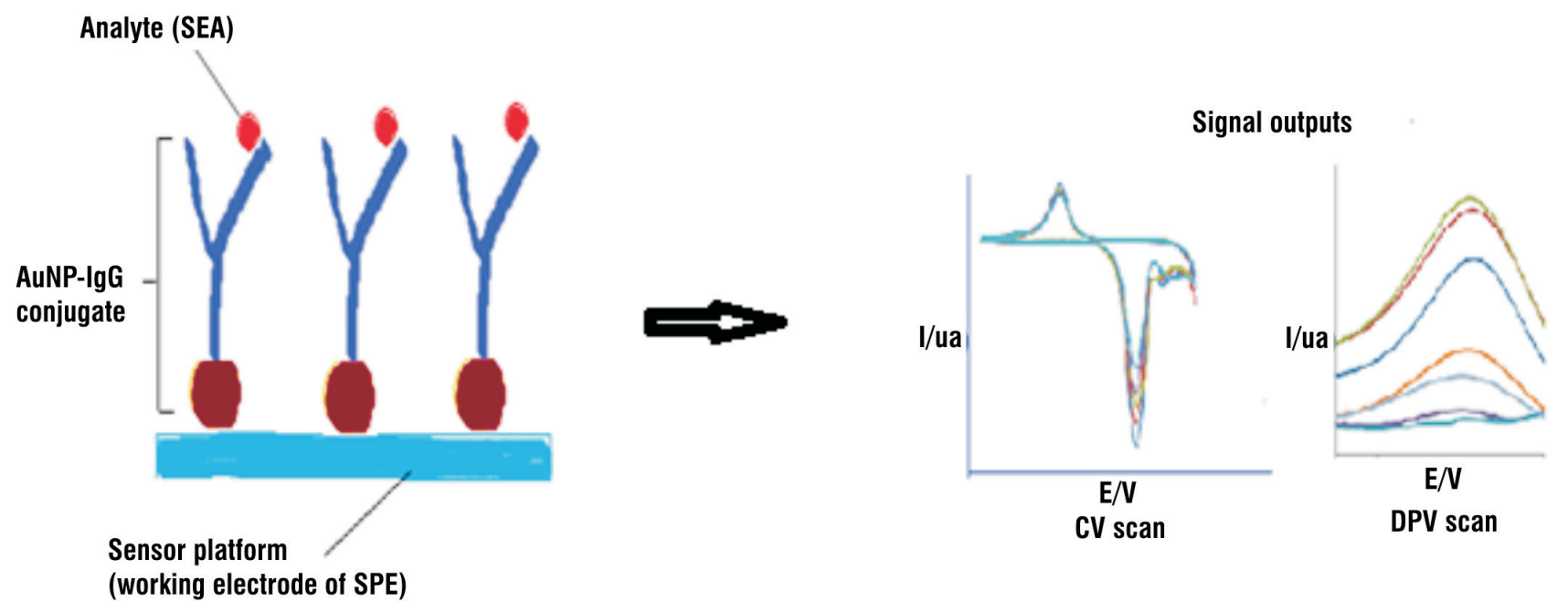

Scheme 1

The immunosensor protocol for analyte determination based on direct determination of the electrochemical signal obtained after Ab-Ag interaction. The peak currents are found to be proportional to the concentration of SEA. ${ }^{21}$ 
(a)

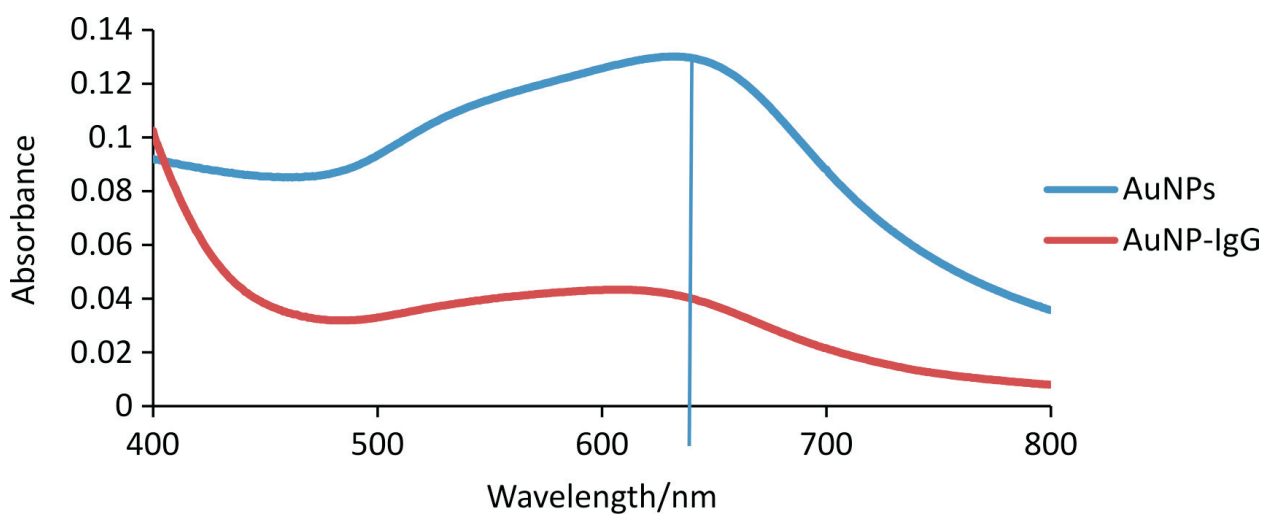

(b)

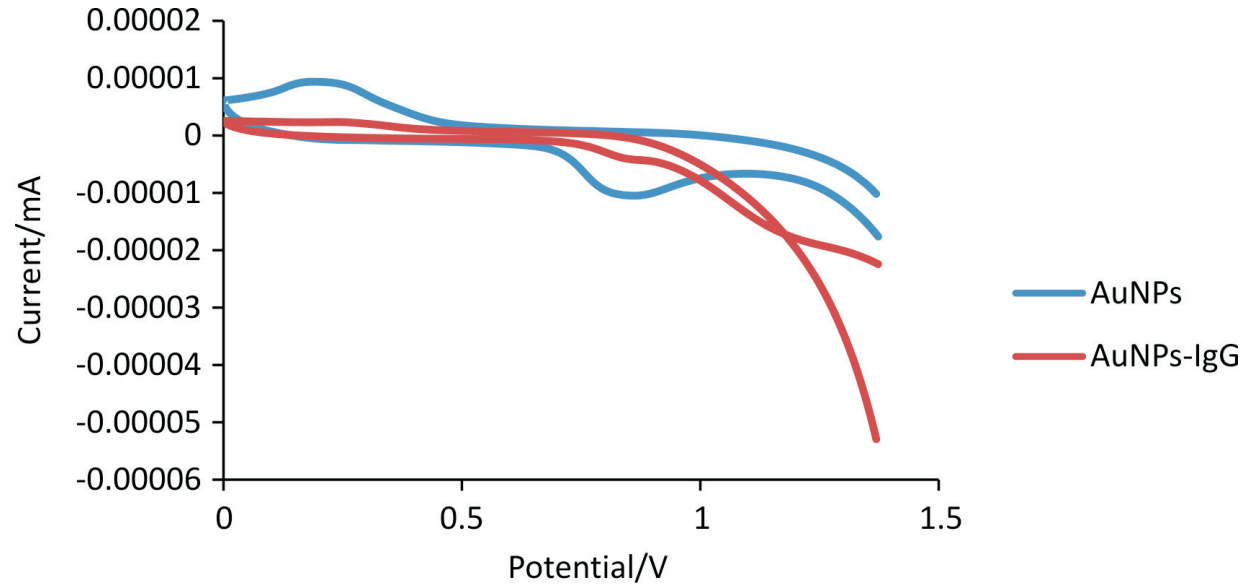

Figure 2 (a) UV spectrum of $10 \mu$ l AuNPs and AuNP-IgG conjugate in 0.01 M PBS, ${ }^{19} \mathrm{n}=3$. (b) Cyclic voltammograms of AuNPs and AuNP-IgG conjugate immobilized on GCE electrodes for $1 \mathrm{~h}^{19}, \mathrm{n}=3$.

that a new compound consisting of the nanoparticle and the antibody has been formed.

\subsection{Study of the Nanoparticle-anti-bilharzia Conjugate and the Soluble Egg Antigen (SEA) Interaction}

The nano-biosensor was characterized by studying the interaction of the nanoparticle-anti-bilharzia conjugate with the SEA and this was done using cyclic voltammetry and differential pulse voltammetry.

\subsubsection{Cyclic Voltammetry (CV)}

$\mathrm{CV}$ was used to characterize the electrode surface where the nanoparticle-antibody conjugate was immobilized. Oxidizing the gold nanoparticles (AuNPs) in the presence of the rabbit anti-bilharzia antibody formed reactive gold oxides accompanied by electrons which produced redox signal. For screenprinted gold electrodes (Model DS 250BT), the scan indicated anodic and cathodic peaks $920 \mathrm{mV}$ and $325 \mathrm{mV}$, respectively, for gold electrodes. ${ }^{24}$ The peak currents of the anodic and cathodic peaks indicated insignificant changes after several cycles and this indicated the stability as well as the reproducibility of the AuNPs-rabbit anti-bilharzia conjugate. After the incubation of the conjugate modified electrode with different concentrations of standards of SEA prepared in PBS buffer ( $\mathrm{pH}$ 7.4), the results indicated a biospecific molecular recognition, forming an antibody-antigen complex evidenced by the change in the redox current $^{25}$ as shown in Fig. 3a. The complex formation on the surface of the electrode led to an insulation of the surface toward the redox couple. This insulation mirrored the differences in the effective diffusion of ions to the electrode surface due to the $\mathrm{Ab}-\mathrm{Ag}$ interaction. A calibration curve for the change in the anodic peak current versus the SEA concentration as shown in
Fig. 3b indicated a limit of detection of $8.387 \times 10^{-2} \mathrm{~mL}$ calculated as three times the standard deviation of the blank. To establish that the peak current reduction was due to the antibody-antigen interaction, a control was performed. The control was just a buffer solution without incubation of the SEA. The peak current showed insignificant change and this indicated that the recorded current decrease was due to the SEA and its antibody interaction.

For screen-printed gold electrodes (Model DS 220BT) the scans revealed an anodic and a cathodic peak at $950 \mathrm{mV}$ and $325 \mathrm{mV}$, respectively, which was similar to the observation made for Model DS 250BT. As expected, the peak currents recorded indicated no significant changes, indicating the stability and reproducibility of the conjugate. Varying standards of SEA were then incubated with the conjugate and CV scans conducted (Fig. 4a). A calibration curve for the anodic peak current versus the SEA concentration as shown in Fig. $4 \mathrm{~b}$ revealed a limit of detection of $5.45 \times 10^{-2} \mathrm{ng} \mathrm{mL}^{-1}$ calculated as three times the standard deviation of the blank.

\subsubsection{Differential Pulse Voltammetry}

Differential pulse voltammetry (DPV) was used to estimate the linear range, limit of detection and individual quantification of SEA in various real samples since it has a much higher current sensitivity and selectivity than $\mathrm{CV}^{26}$ It was noted that the peak current (Ip) at $-0.11 \mathrm{~V}$ was decreasing with the increase in the concentration of SEA. This was because the complex formed at the surface of the electrode led to an insulation of the electrode surface towards the redox couple reflecting the differences in the effective diffusion of ions to the electrode surface due to the $\mathrm{Ab}-\mathrm{Ag}$ complex interaction. The extent of insulation was found to be dependent on the concentration of SEA used and it 
(a)

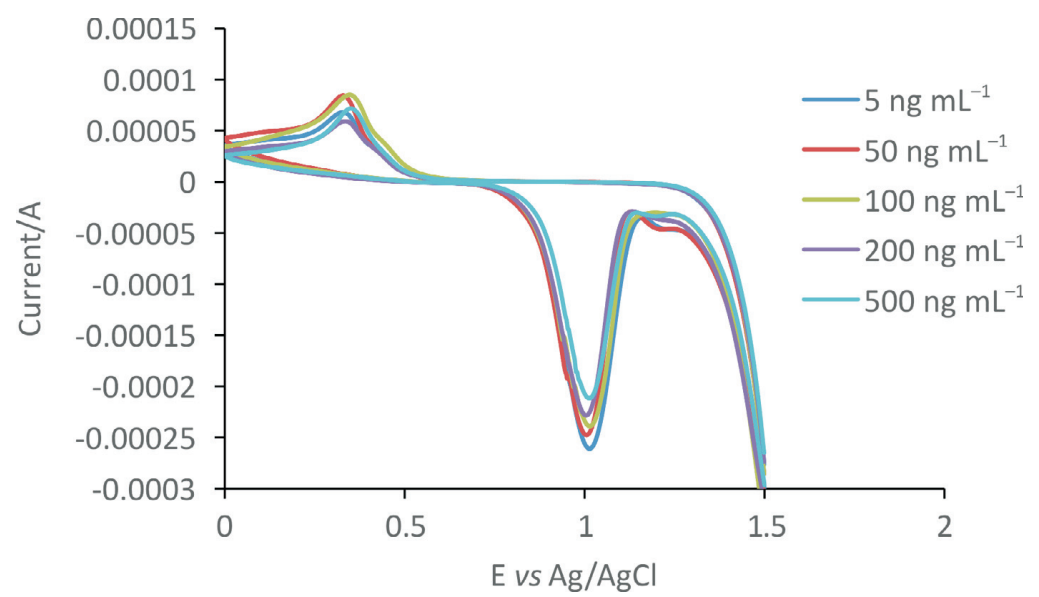

(b)

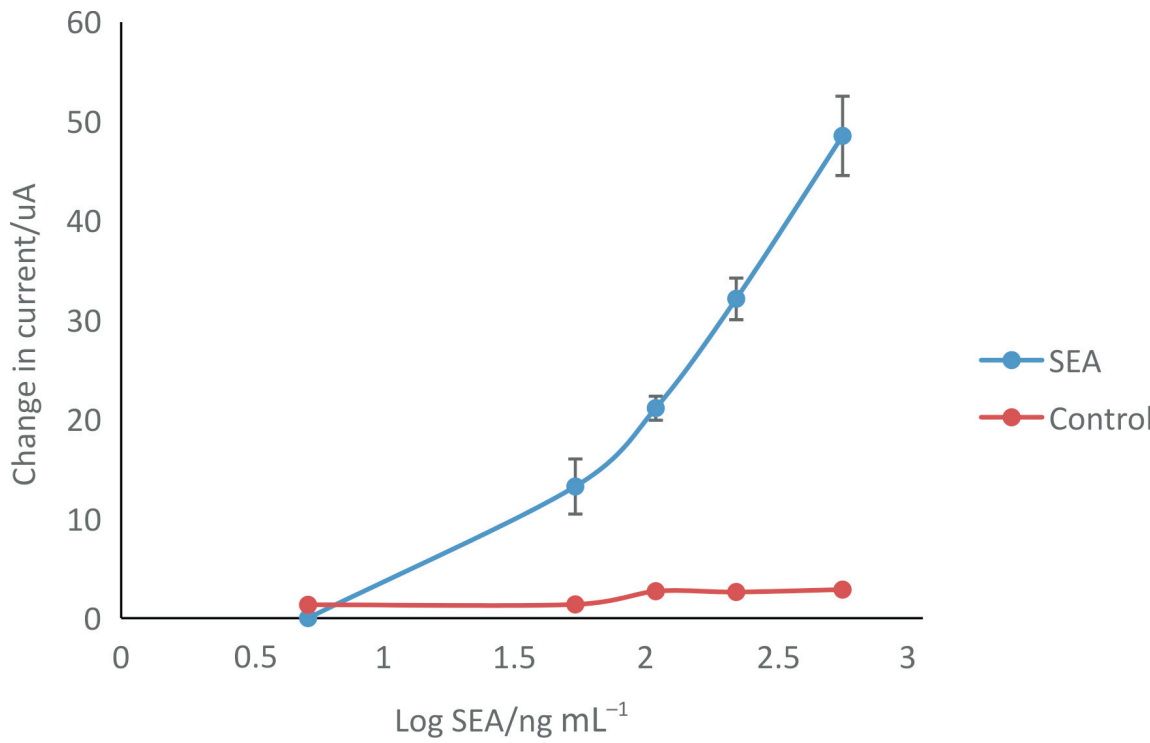

Figure 3 (a) Representative cyclic voltammograms obtained after incubating the AuNPs-rabbit anti-bilharzia antibody modified screen-printed gold electrodes (Model DS 250BT) in varying concentrations of SEA, $n=3$. (b) SPE (Model DS 250BT) nano-biosensor CV calibration curve for the change in anodic peak currents versus log of concentration of SEA, $n=3$.

reduced in the intensity of the peak currents as the concentration was increased (Fig. 5a). The peak currents were noted to decrease with the increase in concentration of SEA incubated with the conjugate modified SPE. For SPE (Model DS 220BT), the peak current appeared at $-0.274 \mathrm{~V}$ (Fig. 5a). A plot of concentration of SEA against the change in peak currents gave detection limits of $3.64 \times 10^{-5} \mathrm{ng} \mathrm{mL}^{-1}$ calculated as three times the standard deviation of the blank (Fig. 5b).

\subsection{Determination of Schistosomiasis mansoni SEA in Human Faecal Material}

The performance of the biosensor was validated using human

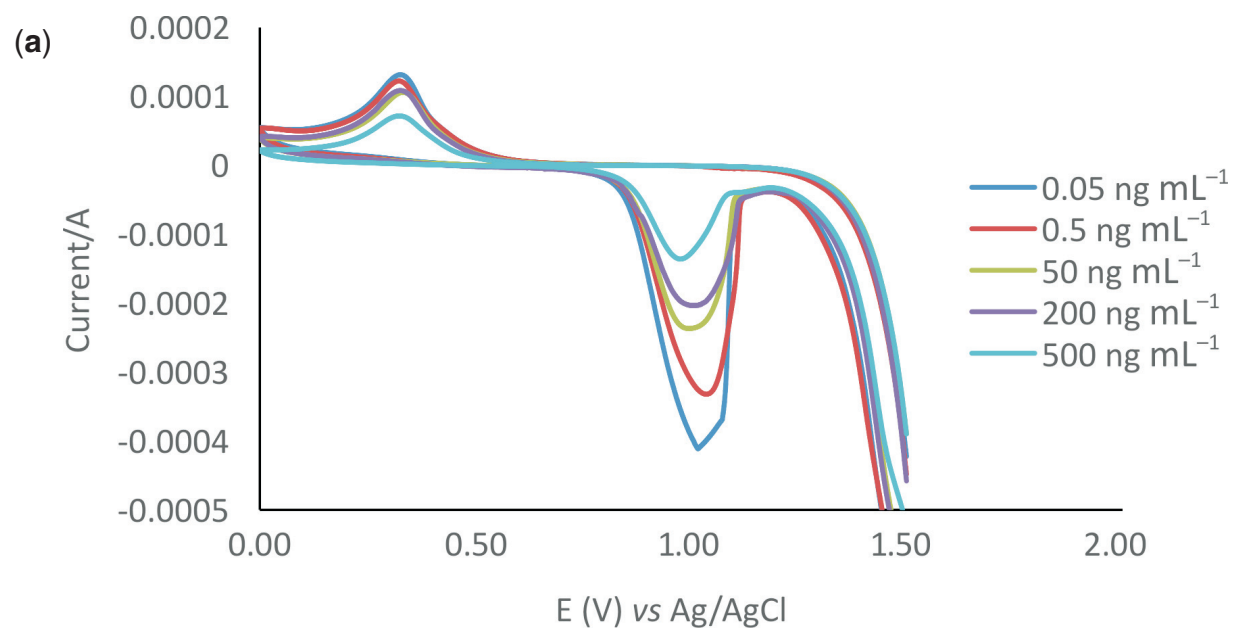

Figure 4 (a) Representative cyclic voltammograms obtained after incubating the AuNPs-rabbit anti-bilharzia antibody modified screen-printed gold electrodes (Model DS 220BT) in varying concentrations of SEA, $n=3$. (Continued on p. 132.) 
(b)

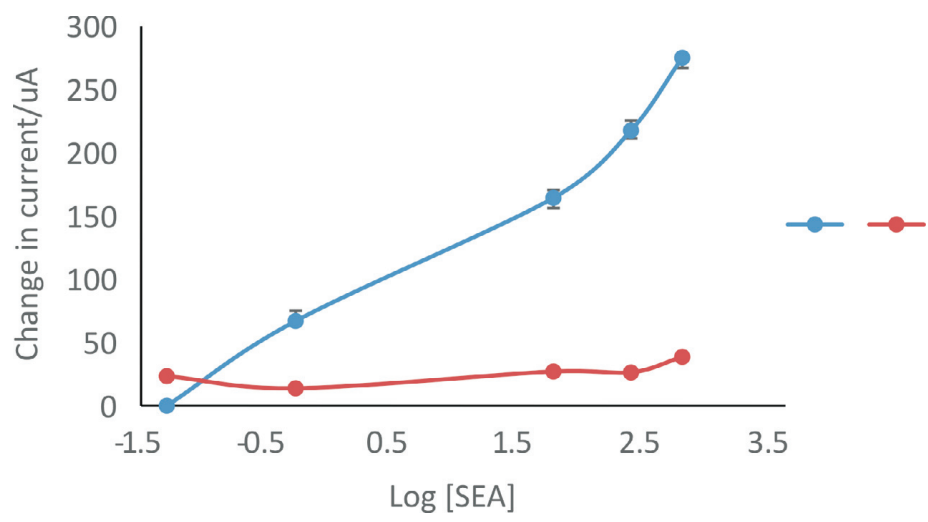

Figure 4 (Continued) (b) SPE (Model DS 220BT) nano-biosensor CV calibration curve for the change in anodic peak currents verseslog of concentration of SEA, $\mathrm{n}=3$.
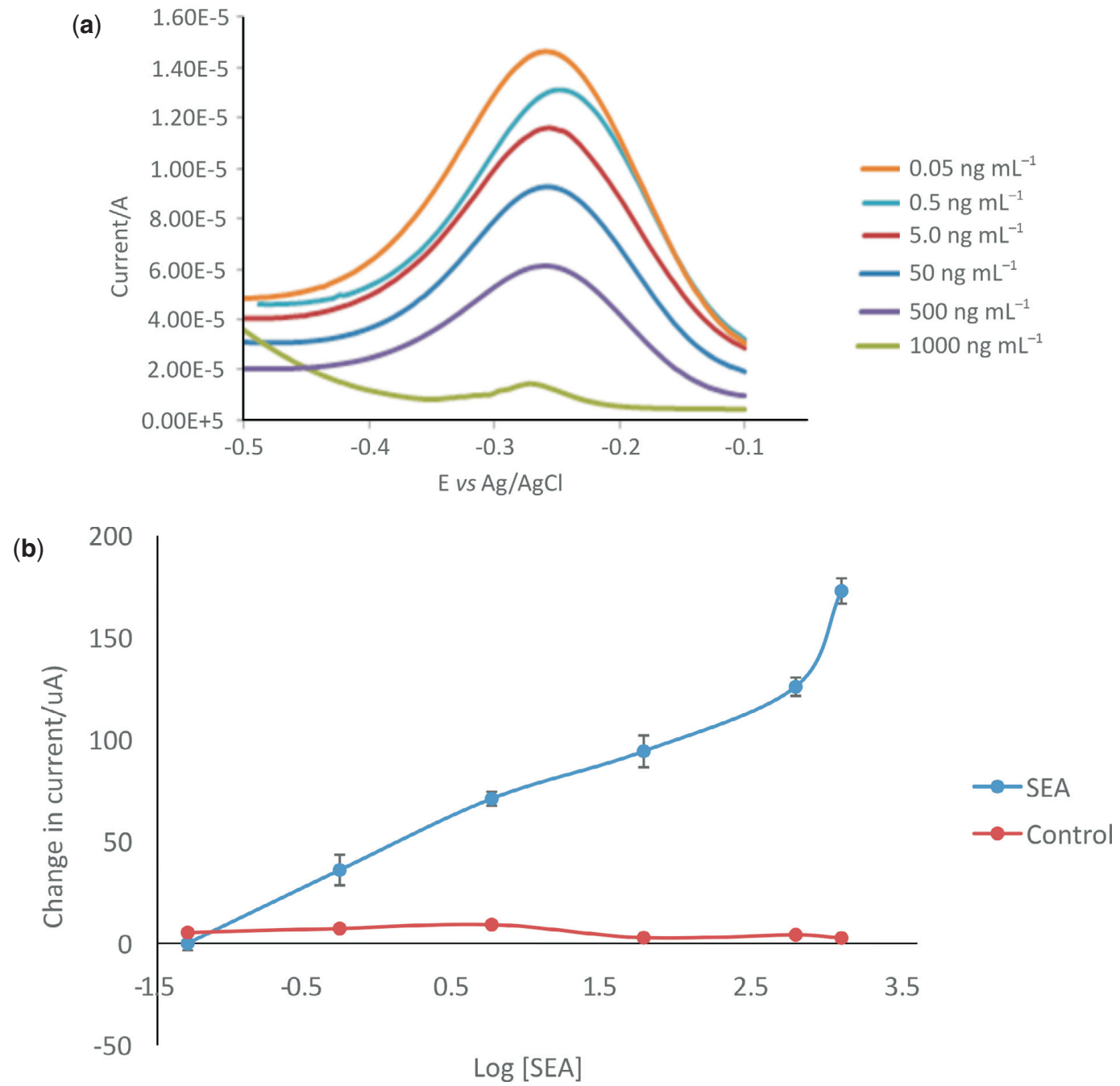

Figure 5 (a) Representative differential pulse voltammograms obtained after incubating the AuNPs-rabbit anti-schistosome antibody modified screen-printed gold electrodes (Model DS 220BT) in varying concentrations of SEA, $n=3$. (b) SPE (Model DS 220BT) nano-biosensor DPV calibration curve using a plot of change in peak currents versus the log of the concentration of SEA. LOD $=3.64 \times 10^{-5} \mathrm{ng} \mathrm{mL} L^{-1}, \mathrm{n}=3$.

faecal samples suspected to be infected with a species of Schistosoma. The samples were diluted with $0.01 \mathrm{M}$ PBS $(\mathrm{pH}=$ 7.4) buffer. $10 \mathrm{ml}$ of the mixture was vortexed for $5 \mathrm{~min}$ to break the eggs and release miracidia. This was then centrifuged for $1 \mathrm{~h}$ at $3000 \mathrm{rpm}$ to separate the debris. $20 \mu \mathrm{l}$ of the supernatant was diluted to a factor 4 and analyzed using both CV and DPV with the nano-biosensor. The actual concentration of the sample was determined from the equation of the regression line obtained after running a series of standards of SEA.

\subsection{Reproducibility and Selectivity Tests}

The reproducibility of the nano-biosensor based on the Ab-Ag interaction in the presence of SEA was investigated and only a small difference in both CV and DPV peak current was observed. The relative standard deviation was less than $10 \%$ when five repeats with different SPE electrodes and different times were measured, indicating that the reproducibility of the nano-biosensor is acceptable in these experiments. $20 \mu \mathrm{l}$ of a sample whose concentration is known was spiked with different 
concentrations of standards of SEA and then analyzed with the nano-biosensor using the two techniques. The concentrations of SEA standards spiked in the sample were 0.5, 5.0 and $50.0 \mathrm{ng} \mathrm{mL}^{-1}$ after constructing calibration curves for SEA standard solutions between $5.0 \times 10^{-2} \mathrm{ng} \mathrm{mL}^{-1}$ and $5.0 \times 10^{2} \mathrm{ng} \mathrm{mL}^{-1}$. The response from the nano-biosensor of each of spiked sample was then compared to the response of the standard of SEA concentration and the values used to calculate the recovery. This showed an increase in the response as a result of increase in concentration. After subtracting the blank, the recoveries were then calculated using the following equation:

$$
\% \text { Recovery }=\frac{\text { Response signal of spiked sample }}{\text { Response signal of standard }} \times 100
$$

The percentage recoveries were found to be between 75 and $92 \%$ for CV with an average of $80.34 \%$ and between 71 and $96 \%$ with an average of $85.2 \%$ for DPV. These averages indicate a good recovery and may imply that there were less or no sources of interference in the stool samples. Therefore this nano-biosensor can be used to detect SEA in faecal material of patients with Schistosoma mansoni.

\section{Conclusion}

We have developed and validated the chemistry of a nanobiosensor for the detection of bilharzia in real samples using screen-printed electrodes. The nanobiosensor was tested and validated using real stool samples. A detection range of $1.13 \times$ $10^{1} \mathrm{ng} \mathrm{mL}^{-1}$ to $2.3 \times 10^{3} \mathrm{ng} \mathrm{mL}^{-1}$ of bilharzia antigen in stool samples and with a limit of detection of $8.3887 \times 10^{-2} \mathrm{ng} \mathrm{mL}^{-1}$ was obtained indicating the potential of the strips to be used for point-of-care devices to detect bilharzia antigen in real samples. A reproducibility of $1.5 \%$ was also obtained. This nano-biosensor can be fabricated to nano-kits which can be distributed to bilharzia-endemic areas in Kenya like Nyanza and Mwea regions to facilitate early diagnosis and treatment of bilharzia.

\section{Acknowledgements}

The authors would like to thank the following institutions: Chemistry Department, Kenyatta University, Nairobi, Kenya; School of Pharmacy and Health Sciences, United States International University-Africa, Nairobi, Kenya; Multimedia University of Kenya, Nairobi, Kenya; and Centre for Biotechnology Research \& Development, Kenya Medical Research Institute, Nairobi, Kenya, for the facilities they provided for this work. This work was supported by Grand Challenges Canada (GCC) grant number 0426-01 and the Third World Academy of Sciences (TWAS) grant number 13-115 RG/CHE/AF/AC_I.

Earlier version of this work was presented at the 8th International Conference of the Kenya Chemical Society held at the University of Nairobi, Kenya, and as a poster at the Pittcon Conference held in Louisiana, New Orleans, USA, March 2015.

\section{${ }^{8}$ ORCID iD}

N. Noah:

orcid.org/0000-0002-2285-4475

\section{References}

1 T. Kariuki, R. Phillips, S. Njenga, O.F. Olesen, P.R. Klatser, R. Porro, S. Lock, M.H. Cabral, M. Gliber and D. Hanne, Research and capacity building for control of neglected tropical diseases: the need for a different approach, PLOS Negl. Trop. Dis., 2011, 5, e1020.

2 a) WHO, Vol. 15, February, 2010 edn., World Health Organization, 2010, pp. 25-98; b) J. Utzinger, G. Raso, S. Brooker, D. De Savigny, M. Tanner, N. Ørnbjerg, B. Singer and E. N'goran, Schistosomiasis and neglected tropical diseases: towards integrated and sustainable control and a word of caution, Parasitol., 2009, 136, 1859-1874.

3 a) L. Chitsulo, P. Loverde and D. Engels, Schistosomiasis, Nat. Rev.
Microbiol., 2004, 2, 12-13; b) B. Gryseels, K. Polman, J. Clerinx and L. Kestens, Human schistosomiasis, Lancet, 2006, 368, 1106-1118; c) A.G. Ross, D. Vickers, G.R. Olds, S.M. Shah and D.P. McManus, Katayama syndrome, Lancet Infect. Dis., 2007, 7, 218-224; d) A.G.P. Ross, P.B. Bartley, A.C. Sleigh, G.R. Olds, Y. Li, G.M. Williams and D.P. McManus, Schistosomiasis, New Engl. J. Med., 2002, 346, 1212-1220.

4 a) B. Ayele, B. Erko, M. Legesse, A. Hailu and G. Medhin, Evaluation of circulating cathodic antigen (CCA) strip for diagnosis of urinary schistosomiasis in Hassoba school children, Afar, Ethiopia, Parasite, 2008, 15, 69-75; b) G.J. van Dam, J.H. Wichers, T.M.F. Ferreira, D. Ghati, A. van Amerongen and A.M. Deelder, Diagnosis of schistosomiasis by reagent strip test for detection of circulating cathodic antigen, J. Clin. Microbiol., 2004, 42, 5458-5461; c) L. van Etten, C.C. Folman, T.A. Eggelte, P.G. Kremsner and A.M. Deelder, Rapid diagnosis of schistosomiasis by antigen detection in urine with a reagent strip, J. Clin. Microbiol., 1994, 32, 2404-2406.

5 Rapid, For qualitative detection of bilharzia (Schistosoma) (25 Diagnostic tests), http://www.rapid-diagnostics.com/downloads/RMD\% 20Pamphlet\%20Just\%2030.09.08.pdf

6 J. Utzinger, M. Booth, E.K.N'Goran, I. Müller, M. Tanner and C. Lengeler, Relative contribution of day-to-day and intra-specimen variation in faecal egg counts of Schistosoma mansoni before and after treatment with praziquantel, Parasitol., 2001, 122, 537-544.

7 C.D. Mathers, M. Ezzati and A.D. Lopez, Measuring the burden of neglected tropical diseases: the global burden of disease framework, PLOS Negl. Trop. Dis., 2007, 1.

8 a) C.-C. Lin, L.-C. Chen, C.-H. Huang, S.-J. Ding, C.-C. Chang and H.-C. Chang, Development of the multi-functionalized gold nanoparticles with electrochemical-based immunoassay for protein A detection, J. Electroanal. Chem., 2008, 619-620, 39-45; b) M.T. Pooja, V. Komal, A.D. Vida and R.S. Shree, Functionalized gold nanoparticles and their biomedical applications, Nanomater., 2011, 1, 31-63; c) M. Yang, Y. Kostov, H.A. Bruck and A. Rasooly, Gold nanoparticle-based enhanced chemiluminescence immunosensor for detection of staphylococcal enterotoxin B (SEB) in food, Int. J. Food Microbiol., 2009, 133, 265-271.

9 a) P. Baptista, E. Pereira, P. Eaton, G. Doria, A. Miranda, I. Gomes, P. Quaresma and R. Franco, Gold nanoparticles for the development of clinical diagnosis methods, Anal. Bioanal. Chem., 2008, 391, 943-950; b) D.A. Healy, C.J. Hayes, P. Leonard, L. McKenna and R. O'Kennedy, Biosensor developments: application to prostate-specific antigen detection, Trends Biotechnol., 2007, 25, 125-131; c) Y. Wan, Y. Su, X. Zhu, G. Liu and C. Fan, Development of electrochemical immunosensors towards point of care diagnostics, Biosens. Bioelectron., 2013, 47, 1-11; d) M. Dequaire, C. Degrand and B. Limoges, An electrochemical metalloimmunoassay based on a colloidal gold label, Anal. Chem., 2000, 72, 5521.

10 a) X. Cao, Y. Ye and S. Liu, Gold nanoparticle-based signal amplification for biosensing, Anal. Biochem., 2011, 417, 1-16; b) D. Liu, W. Chen, J. Wei, X. Li, Z. Wang and X. Jiang, A highly sensitive, dual-readout assay based on gold nanoparticles for organophosphorus and carbamate pesticides, Anal. Chem., 2012, 84, 4185-4191.

11 a) G. Liu and Y. Lin, Nanomaterial labels in electrochemical immunosensors and immunoassays, Talanta, 2007, 74, 308-317; b) K. Omidfar, F. Khorsand and M.D. Azizi, New analytical applications of gold nanoparticles as label in antibody based sensors, Biosens. Bioelectron., 2013, 43,336-347; c) M.S. Szymanski and R.A. Porter, Preparation and quality control of silver nanoparticle-antibody conjugate for use in electrochemical immunoassays, J Immunol. Methods, 2013, 387, 262-269; d) A. Ambrosi, F. Airo and A. Merkoçi, Enhanced gold nanoparticle based ELISA for a breast cancer biomarker, Anal. Chem., 2009, 82, 1151-1156; e) A. Escosura-Muniz, A. Ambrosi and N. Merkoci, Electrochemical analysis with nanoparticle-based biosystems, Trends Anal. Chem., 2008, 27, 254-260.

12 a) P. Kocbek, N. Obermajer, M. Cegnar, J. Kos and J. Kristl, Targeting cancer cells using PLGA nanoparticles surface modified with monoclonal antibody, J. Control Release, 2007, 120, 18-26; b) K. Cho, X. Wang, S. Nie and D.M. Shin, Therapeutic nanoparticles for drug delivery in cancer, Clin. Cancer. Res., 2008, 14, 1310-1316.

13 S. Zhang, N. Wang, H. Yu, Y. Niu and C. Sun, Covalent attachment of glucose oxidase to an $\mathrm{Au}$ electrode modified with gold nanoparticles for use as glucose biosensor, Bioelectrochem., 2005, 67, 15-22.

14 C. Zhang, Z. Zhang, B. Yu, J. Shi and X. Zhang, Application of the biological conjugate between antibody and colloid Au nanoparticles as 
analyte to inductively coupled plasma mass spectrometry, Anal. Chem., 2002, 74, 96-99.

15 https://www.omicsonline.org/

16 O.D. Renedo, M. Alonso-Lomillo and M.A. Martínez, Recent developments in the field of screen-printed electrodes and their related applications, Talanta, 2007, 73, 202-219.

17 M. Pravda, C.O'Meara and G.G. Guilbault, Polishing of screen-printed electrodes improves IgG adsorption, Talanta, 2001, 54, 887-892.

18 S. Kröger, A.P. Turner, K. Mosbach and K. Haupt, Imprinted polymer-based sensor system for herbicides using differential-pulse voltammetry on screen-printed electrodes, Anal. Chem., 1999, 71, 3698-3702.

19 J. Odundo, M.N. Naumih, D. Andala, J. Kiragu, M. Ndikau, G. Kimani and J. Mwatha, Spectro-electrochemical characterization of anti-schistosoma-gold nanoparticle conjugate for use in immunoassays, J. Kenya Chem. Soc., 2016, 9, 35-45.

20 J. Clerinx, L. Cnops, T. Huyse, E. Tannich and M. Van Esbroeck, Diagnostic issues of acute schistosomiasis with Schistosoma mekongi in a traveler: a case report, J. Travel Med., 2013, 20, 322-325.

21 M. Arruebo, M. Valladares and A. Gonzalez-Fernandez, Antibody- conjugated nanoparticles for biomedical applications, J. Nanomater., 2009, 2009.

22 E.K. Payne, K.L. Shuford, S. Park, G.C. Schatz and C.A. Mirkin, Multipole plasmon resonances in gold nanorods, J. Phys. Chem. B, 2006, 110, 2150-2154.

23 D.L. Thorek, D.R. Elias and A. Tsourkas, Comparative analysis of nanoparticle-antibody conjugations: carbodiimide versus click chemistry, Mol. Imaging, 2009, 8, 221-229.

24 a) L. Wang and E. Wang, Direct electron transfer between cytochrome $\mathrm{c}$ and a gold nanoparticles modified electrode, Electrochem. Commun., 2004, 6, 49-54; b) X. Dai, O. Nekrassova, M.E. Hyde and R.G. Compton, Anodic stripping voltammetry of arsenic (III) using gold nanoparticle-modified electrodes, Anal. Chem., 2004, 76, 5924-5929.

25 N.M. Noah, O. Marcells, A. Almalleti, J. Lim and O.A. Sadik, Metal enhanced electrochemical cyclooxygenase-2 (COX-2) sensor for biological applications, Electroanal., 2011, 23, 2392-2399.

26 K.T. Kawagoe, J.B. Zimmerman and R.M. Wightman, Principles of voltammetry and microelectrode surface states, J. Neurosci. Methods, $1993,48,225-240$. 